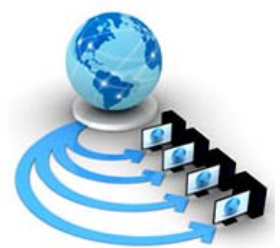

International Journal of Advanced Research in Computer Science

RESEARCH PAPER

\author{
Available Online at www.ijarcs.info
}

\title{
A HYBRID ANT COLONY OPTIMIZATION FOR OPTIMAL PATH PLANNING IN WIRELESS SENSOR NETWORK
}

\author{
Pallavi \\ M.tech(CSE), \\ DCRUST,India
}

\author{
Mr.Sanjeev Indora \\ A.P, CSE Dept., \\ DCRUST,India
}

\begin{abstract}
A Wireless Sensor System incorporates a pack of nodes associated with ordinarily low usefulness. They work with other all the things considered to execute acknowledging assignments amongst acknowledged environment. An undetectable identifier group may incorporate one specific or many deplete nodes to gather implicit know how apart trade that to a main system anyhow storage space technique. A standout amongst the most issues experienced in these systems, is finding the protected route amongst starting point and ending point with limiting invaders impacts. As energy and bandwidth is the main constraint in any WSN so there is no requirement to pass the information through all the nodes. Only the required nodes take part in communication. In the proposed work we have used Ant colony optimization algorithm. We have considered various parameters in our work on the basis of which we have computed our algorithm. Simulation results are carried out using MATLAB. The results are shows in the graphical as well as in numerical forms.
\end{abstract}

Keywords: Ad-Hoc, WSN, AODV, route repairing etc.

\section{INTRODUCTION}

An ad-hoc network [1] is the cooperative engagement of a collection of mobile nodes without the required intervention of any centralized access point or existing infrastructure. It typically refers to any set of networks where all devices have equal status on a network and are free to associate with any other ad hoc network devices in link range. Very often, ad hoc network refers to a mode of operation of IEEE 802.11 wireless networks [2].

\subsection{Types of Ad-hoc Network}

\subsubsection{Wireless Mesh Network \\ 1.1.2 Wireless Sensor Network \\ 1.1.3 Mobile Ad-hoc network}

\subsection{Wireless Sensor Network}

The WSN is built of "nodes" [3] - from a few to several hundreds or even thousands, where each node is connected to one (or sometimes several) sensors. Each such sensor network node has typically several parts: a radio transceiver with an internal antenna or connection to an external antenna, a microcontroller, an electronic circuit for interfacing with the sensors and an energy source, usually a battery or an embedded form of energy harvesting. Sensors [4] have been used in precision agriculture for years. They are used in convergence with other technologies like global positioning system (GPS), Geographic information system (GIS), miniaturized computer components and automatic control and remote sensing.

The basic requirements of WSN are as follows [5]:

- Scalability- WSN must be capable of being easily expanded or upgraded on demand.

-Reliability- WSN must be worth trusting, it should provide what is needed for the user.

- Responsiveness- WSN should quickly react in the desired or positive way.
- Mobility- WSN must be able to move from one place to another.

- Power efficiency- WSN must be power efficient.

One of the biggest weakness of large WSN is absence of life span as it is extremely hard to replace the sensor nodes which are organized in the unfriendly areas. This may be imaginable that within 15-20 years the whole world will come under wireless sensor networks that access via internet [6].

WSN nodes are energy, computation, and memory restricted. Major importance is given to Energy consumption in WSNs and some techniques and hardware were considered with energy efficiency or energy alertness as a centre point of interest.

There are two techniques to save energy which focuses on two systems: the networking subsystem and the sensing subsystem. In networking subsystem, the basic criteria for energy supervision of every node is calculated alongside the plan of networking protocols. In sensing subsystem, the frequency of energy-expensive data samples is reduced by different methods [7].

\section{LITERATURE SURVEY}

In the year 2006, HaoMei YantaoTian and LinanZum [8] worked on Ant colony optimization and artificial potential field were utilized individually as worldwide way arranging and nearby way arranging strategies in the paper. A few adjustments were made to suit deep ant colony optimization to way arranging. Pheromone produced by ant colony optimization was additionally used to keep artificial potential field from getting local minimum.

Vinay Kumar Singh and Vidhusi Sharma [9] played out a work, "Elitist Genetic Algorithm Based Energy Efficient Routing Scheme for Wireless Sensor Networks". This paper utilized a forward address based most limited way routing in the system. Genetic calculation with elitism idea is utilized 
to acquire energy productive routing by limiting the way length and subsequently expanding the life of the system.

In the year 2009, JiannHorng Lin and Li Ren Huang [10] worked on swarm knowledge and violent elements for learning. Creators tended to this issue by considering the issue of way making arrangements for mobile robots. In this paper, Authors proposed another way to deal with the issue of way getting ready for mobile robots in light of an enhanced artificial honey bee settlement advancement joined with chaos.

In the year 2013, Priyadharshini. T.; Arunachalam. Ar[11] proposed Genetic algorithm which is a problem solving assessment method for solving the ideal path routing problem. Fitness function is evaluated for each candidate by the use of genetic algorithm. The fitness capacity of insignificant cost limit and data transmission measurements to ideal way for begin and end nodes in ad-hoc networks. The process is executed in JAVA.

In the year 2009, M. bheemalingaiah et al. [12] played out a work in which the creators considered the multipath routing algorithm to discover the way which brings about least cost as far as distance. The creator proposed the strategy to upgrade the consumed energy by choosing a practical way from source to goal.

In the year 2010, Hui Cheng and Shengxiang Yang [13] proposed to utilize multi-population GAs with foreigners plan to take care of the dynamic SP issue in MANETs which is the agent of new era remote systems. The trial comes about demonstrate that the proposed GAs can rapidly adjust to the natural changes (i.e., the system topology change) and create great arrangements after each change.

\section{Methodology}

The point of this section is to talk about around a calculation for localization of nodes in a sensor network. The calculation ought to be conveyed and executed in singular nodes. Since the calculation ought to be keep running in singular sensor nodes, the arrangement must be moderately basic, and request constrained assets. The objective is to have the capacity to position nodes with a given precision, or to group nodes as being "non-localizable". The execution of confinement calculations will rely on upon basic sensor organize parameters, for example, the radio range, the thickness of nodes, the anchor to-hub proportion, and it is critical that the arrangement gives sufficient execution over a scope of sensible parameter esteems.

\subsection{Ant colony optimization (ACO)}

The ACO (Ant Colony Optimization) heuristic is motivated by the genuine ant conduct (figure 3.1). It solves the problem of finding optimal path from source to destination on basis of real ant behavior. In starting ants move randomly to find source. When food source found, ants move back to colony. Ants leaving pheromones while moving back to colony that shows path for food. Other ants follow the same path to reach on food source. Pheromone makes a stronger path as many ants move on same path. The amount of pheromone is deposited, which may depend on the quantity and quality of the food. After some time when food sources decreases, the path has no longer pheromones. [14] It finds optimal and shortest path for data transmission in wireless sensor networks. ACO applied only where source and destination are predefined. It not works well if paths are not symmetric. [15]

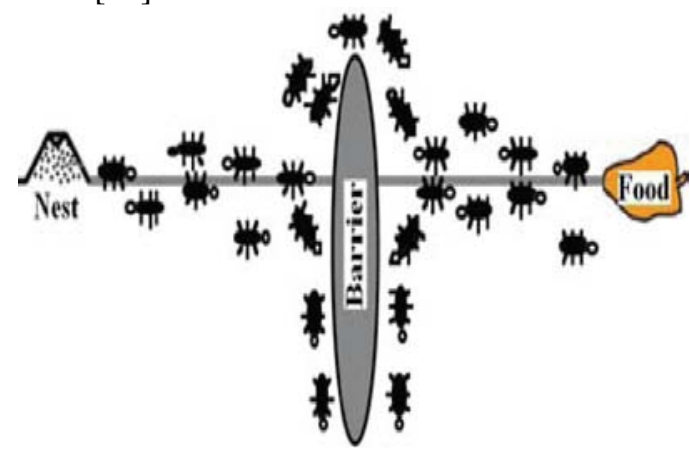

Fig3.1: Real ANT behavior in finding the path

The objective of this work is towards improving the Quality of service of the wireless sensor network by minimization of power consumption in wireless network which has been done by considering various different parameters. The power consumption problem has been reduced to a nonlinear problem with constraints. The mathematical problem for this work is represented as:

objf $=\sum P_{T X} \times U R$

Keeping these constraints into consideration, Ant colony algorithm looks for optimal edge which consumes least power in transmission. The proposed work is divided into two sections. In one part route selection is done and in another part channel allocation to each route is done using Ant optimization algorithm to find the best estimated cost. This route selection is based on minimum distance between active nodes. Route selected by data packet is that which has optimal distance between source and destination.

\section{SIMULATION}

\subsection{Introduction}

The simulations were carried out by using MATLAB as the language that we use to develop the proposed framework. We have used a random ad-hoc network using different number of nodes with the network area of 100 by 100 (sq m).

Ant Colony Optimization for different parameters (distance, Simulated Time, delay etc) and executed for different sizes of network. The results obtained by proposed Approach are presented for comparative analysis with previous approach for different number of nodes. We analyzed that the proposed approach gives a better result in terms of improving Quality of service as compared to existing work. The design network shows that each node can be consider as a source or destination. Hence each node serves as a router in the network which helps in establishing the route from source to destination. 


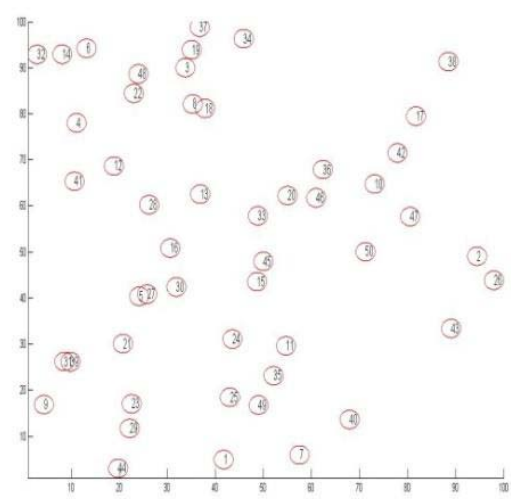

Figure 4.1: Localization of nodes

After the nodes deployment the path has been traced between source node and the destination as shown below.

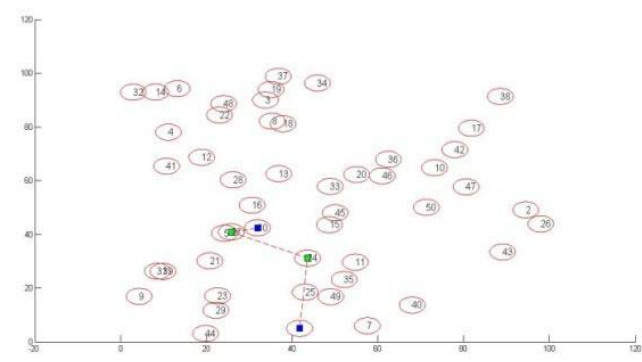

Figure4.2: Route trace

Above figure shows the network simulation model containing 50 nodes. Length versus breadth of the network, the channel $(\mathrm{CH})$ captures the routing information from the initiator (source node) and then sends the data from the source to destination node. In the same manner the simulation has been done with different number of nodes and their experimental graph and result has been noted down which is shown in table 4.1. After that results have been shown in graphical form.

Table4.1 Different parameters with different number of nodes

\begin{tabular}{|l|c|c|c|}
\hline $\begin{array}{l}\text { No. of } \\
\text { Nodes }\end{array}$ & Cost & Delay & Elapsed Time \\
\hline 50 & 318.7767 & $1.8088 \mathrm{e}+05$ & $7.495843 \mathrm{sec}$ \\
\hline 60 & 839.9000 & $1.4316 \mathrm{e}+05$ & $10.338192 \mathrm{sec}$ \\
\hline 80 & 637.1154 & $4.9788 \mathrm{e}+05$ & $18.248635 \mathrm{sec}$ \\
\hline 90 & 468.4115 & $2.3157+05$ & $22.843764 \mathrm{sec}$ \\
\hline 100 & 617.9036 & $1.9627+05$ & $32.999734 \mathrm{sec}$ \\
\hline
\end{tabular}

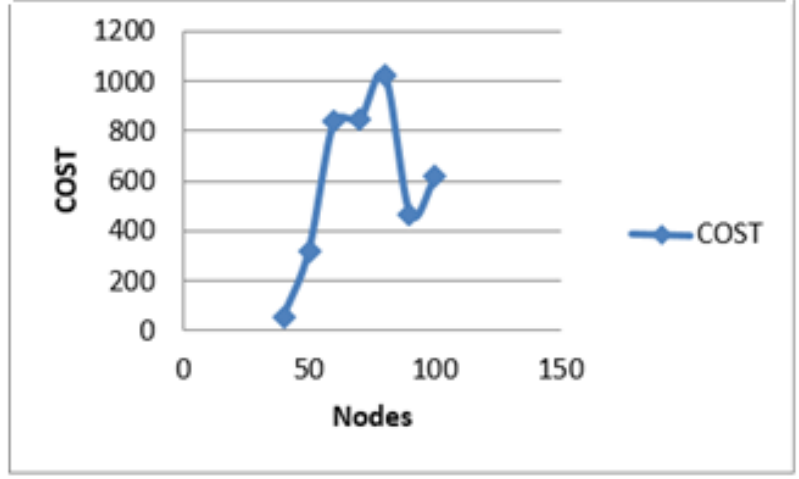

Figure4.3: Nodes v/s Cost

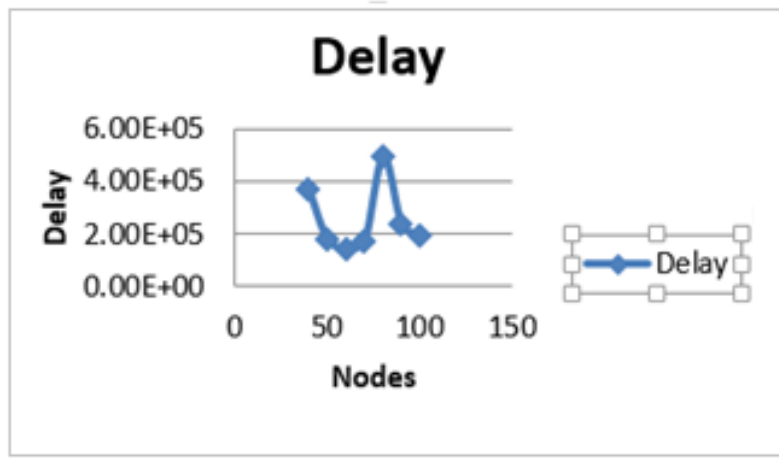

Figure4.4 Nodes v/s Delay

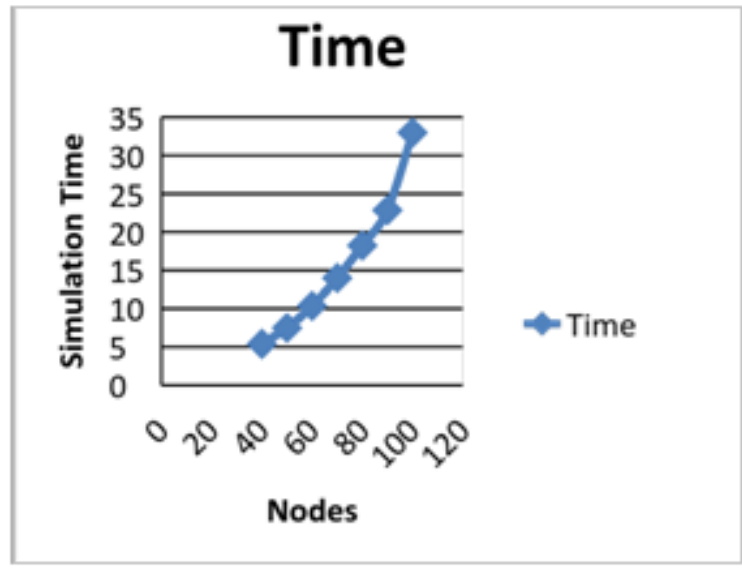

Figure4.5: Nodes v/s Time

\subsection{WSN Network DESIGN: Proposed Model}

The proposed work is about to find the optimal solution to secure the wireless sensor network. The proposed work is to make an energy efficient sensor network which is based on the user entered source and destination. The proposed system will give the benefit in terms of the total cost from source to destination. The network is designed with some defined parameters given as

$\begin{array}{ll}\text { 4.3.1 } \quad \text { Scenario 1 } & \\ \text { Parameter } & \text { Value } \\ \text { Number of Nodes } & 10 \\ \text { Topography Dimension } & 100 \times 100 \mathrm{~m} \\ \text { Traffic Type } & \text { CBR } \\ \text { Topology } & \text { Random } \\ \text { Initial Node } & \text { user defined } \\ \text { Destination Node } & \text { user defined }\end{array}$




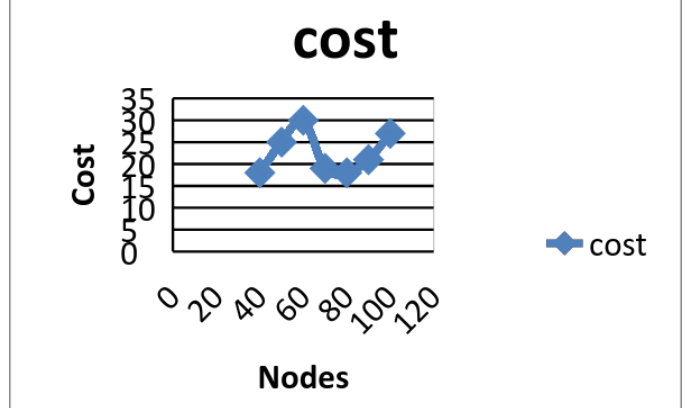

Figure4.6: Plot of Nodes v/s Cost

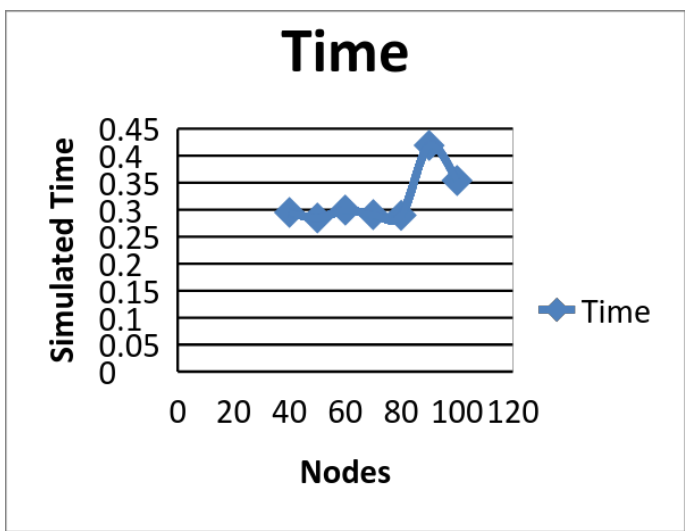

Figure4.7: Nodes v/s Simulation Time

\subsection{COMPARISON GRAPHS}

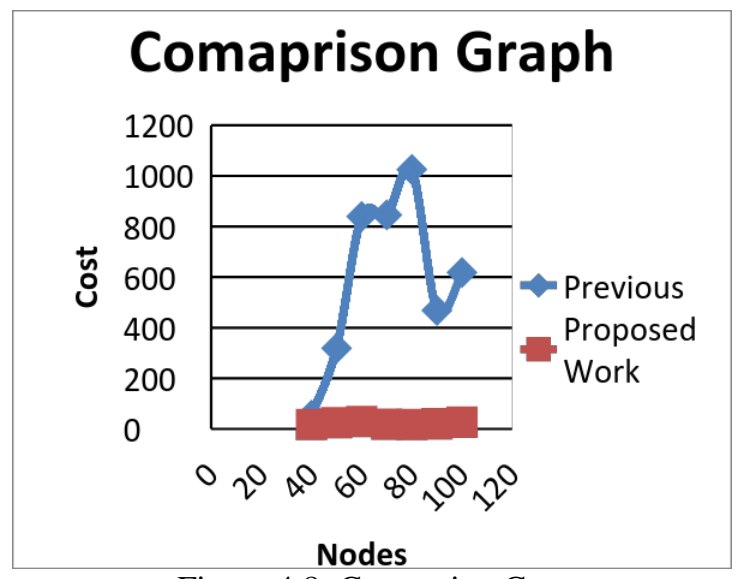

Figure 4.8: Comparing Cost

\section{Comparison Time}

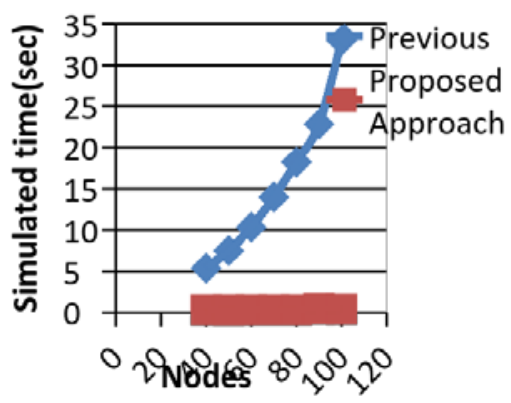

Figure4.9: Comparing Simulated Time

\subsection{SIMULATED PATH}

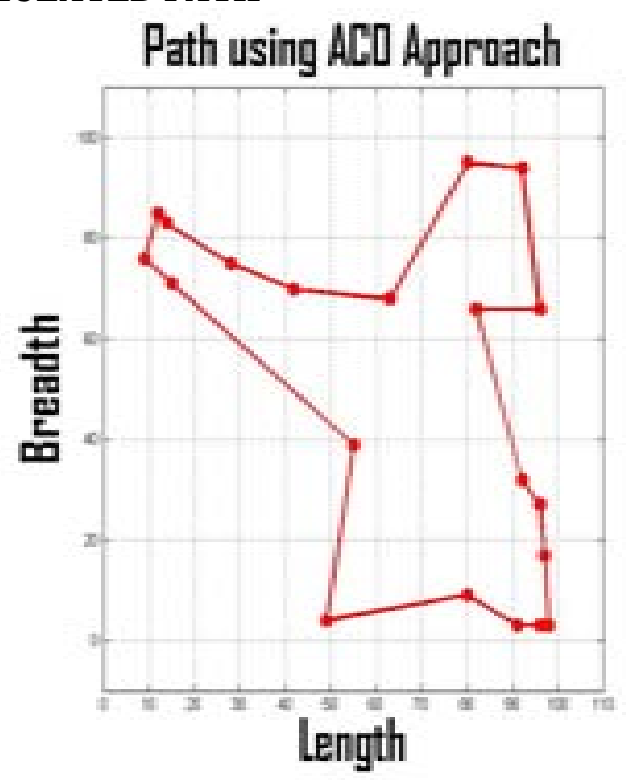

Figure4.10: ACO inspired path

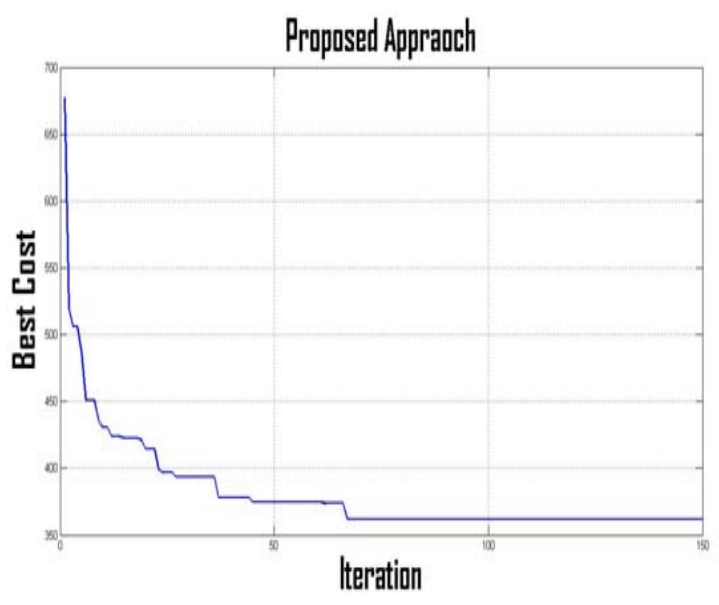

Figure4.11: Best estimated cost

Various graphs have been plotted showing different parameters. As using the proposed approached the Ant colony inspired paths follows a definite pattern from source to destination which results optimized route with best estimated cost.

The effect of using the optimization concept gives the best iterative value instead of the direct generated value. In figure 5.11 the results are optimized in a very well manner using the iterative methodology and the optimization stops as soon as the value reaches to its best i.e362.038 in a very simulated time $\mathbf{9 . 4 9 8 3 1 2}$ seconds whereas the threshold value taken from previous work was 632.5771. The work here is processed for 150 iterations as compared to previous direct methodology. As we can see with the iterations the total distance covered is being reduced. It shows the optimization of result. As the values are continuously decreasing as shown in the figure and finally approaches the best value, this means the route is taken to the best possible way and the route is being optimized. 


\section{CONCLUSION \& FUTURE SCOPE}

As wireless sensor systems turned into a key innovation and are utilized as a part of more mechanical and ecological issues, characterizing a compelling restriction calculation turned into an essential undertaking. The writing review work demonstrated that an ideal calculation couldn't be characterized yet, and in this manner the decision of the reasonable one must be established on the specificities of the circumstances, considering the measure of the system, and in addition the arrangement strategies and the normal outcomes. Proceeding with advance with one of the thoughts recommended that is ACO approach; we studied the impact of various parameters as far as exhibitions. Promote the utilization limitation idea make the system more improved. Henceforth the proposed Ant colony optimization strategy gives better outcomes for the restriction of the nodes in Wireless sensor network.

Sooner rather than later, we trust that in the event that we have the ideal number of nodes grouping, the framework clarity and understandability will increment and will perform better too. In future $\mathrm{ABC}$ calculation might be utilized and examination might be done on similar parameters or with various.

\section{REFERENCES}

[1] Srishti Nanda and Sukhwinder Singh, "BasicStudy of AdHoc Networking”, International Journal of Advanced Research in Computer and Communication Engineering, Vol. 3, Issue 4, April 2014.

[2] Vanita Rani and RenuDhir, "A Study of Ad-Hoc Network: A Review”, International Journal of Advanced Research in Computer Science and Software Engineering,Volume 3, Issue 3, March 2013.

[3] Gagandeep Kaur, "Review paper on Reliability of Wireless Sensor Networks", International Journal of Advanced Research in Computer Engineering \& Technology (IJARCET) Volume 3 Issue 5, May 2014.

[4] ManishaS.Bhende, "Review paper on Topology Issues in wireless Sensor Network", International Journal of Engineering Research and Applications, Vol. 2, Issue 3, May-Jun 2012, pp.1680-1684.

[5] Neha Singh and KamakshiRautela, "Literature Survey on Wireless Sensor Network", International Journal of
Engineering and Computer Science, Volume 5, Issue 8, Aug 2016, Page No. 17544-17548.

[6] KamaldeepKaur ,Aashimasingla” Review paper on Energy Conservation Techniques of Wireless Sensor Networks" International Research Journal of Engineering and Technology (IRJET) Volume: 03 Issue: 10

[7] AashimaSingla, RatikaSachdeva "Review on Energy Conservation in Wireless Sensor Network" International Journal of P2P Network Trends and TechnologyVolume3, Issue2- 2013.

[8] Mei, H., Tian, Y., \&Zu, L. (2006). A hybrid ant colony optimization algorithm for path planning of robot in dynamic environment. International Journal of Information Technology, 12(3), 78-88.

[9] Vinay Kumar Singh and Vidhusi Sharma, "Elitist genetic algorithm based energy efficient routing scheme for wireless sensor networks", International Journal of Advanced Smart Sensor Network Systems, 2(2012), 1521.

[10] Lin, J. H., \& Huang, L. R. (2009, March). Chaotic bee swarm optimization algorithm for path planning of mobile robots. In Proceedings of the 10th WSEAS international conference on evolutionary computing (pp. 84-89). World Scientific and Engineering Academy and Society (WSEAS).

[11] Priyadharshini,T., \&Arunachalam, Ar. (2013). Efficient Genetic Algorithm for Optimal Routing In Ad-hoc Networks. International Journal of Advanced Research in Computer Science and Software Engineering, 3(2), 364367.

[12] Bheemalingaiah, M., Naidu, M. M., Rao, D. S., \&Varaprasad, G. (2009). Energy Aware Node Disjoint Multipath Routing in Mobile Ad-hoc NETWORK. Journal of Theoretical \& Applied Information Technology, 5(4).

[13] Cheng, H., \& Yang, S. (2010). Multi-population genetic algorithms with immigrants scheme for dynamic shortest path routing problems in mobile ad hoc network. Applications of Evolutionary Computation, 562-571.

[14] Sudarshan D Shirkande, Rambabu A Vatti, "ACO based routing algorithm for ad-hoc (WSN, MANET) networks: survey" International Conference on Communication Systems and Network Technologies 2013.

[15]. Bains, V., \& Sharma, K. (2012). Ant colony based routing in wireless sensor networks. International Journal of Electronics and Computer Science Engineering, 1(4), 2516-24. 